\title{
Active Dynamic Tenodesis of Extensor Policis Longus by Palmaris Longus in Deficient Thumb Abduction and Extension
}

\author{
Mohamed A M Gheith", Mohamed A Sebaai \\ Lecturer of orthopedic surgery, Faculty of human medicine, Zagazig University, Egypt
}

"Corresponding Author: Mohamed Abdelaziz MA Ghieth, Lecturer of Orthopedic Surgery, Faculty of Medicine, Zagazig University, Egypt, E-mail: yousufmmkh@gmail.com

\begin{abstract}
Objective: Thumb abduction and extension is one of the most serious and complicated hand function so its absence hinders the grabbing function of other fingers so thumb operations have developed into Active Dynamic Tenodesis of Extensor Policis Longus by Palmaris Longus in deficient thumb abduction and extension rather than static bone operations in succeeding in forming mobile thumb by maintaining the muscular balance via tendon transfer of PL to EPL end to side.
\end{abstract}

Patients and Methods: From 2012 to 2015 reconstructive surgery active dynamic tenodesis was performed on 30 case of deficient thumb abduction and extension. In cases of radial nerve palsy, brachial plexus palsy and spastic cerebral palsy, we use palmaris longus whenever available as it has appropriate line of pull to create abduction - extension and it will not overwhelm thumb flexion. In our cases, we do not detach palmaris longus from its insertion so our aim is to use it as active dynamic tenodesis of thumb abduction and extension when extensor pollicis longus is rerouted and sutured to palmaris longus end to side.

Results: In our study we succeeded in forming mobile thumb by PL transfer and tenodesis to EPL. In our patients, strong lateral pinch on the second middle finger added to maintaining sufficient radial abduction during grabbing were obtained in all patients, the assessment involves physical examination, electromyography. Radiology and diagnostic nerve blocks in CP. $100 \%$ of cases are satisfied, as all patients have good and full thumb function so satisfactory results are achieved.

Conclusion: Our results demonstrate that active dynamic tenodesis of extensor pollicis longus is an effective and simple procedure for restoring thumb extension and abduction in 30 patients with different etiology of deficient thumb abduction and extension.

\section{INTRODUCTION}

Thumb has allowed humans to have superior aptitude for defense, work and dexterity, deficiency of thumb abduction and extension is one of the most complicated disability and is caused by unopposed action of thumb adductors and flexor muscles. Hand function to be effective in grasping, gripping and pinching, the first step is clearance of the thumb from palm by abduction and extension so grasp release , gripping release, pinching release are the first and last motion in hand function. The thumb has exquisite sensibility and is highly mobile structure with well-developed adductor and pronating (thenar) musculature. It is the most important digit of the hand and every effort must be made to preserve its function [1].

Tenodesis is the tendon reinforcement by fixing the tendon to another tendon or a bone periosteum at a proper tension. Dynamic tenodesis is transferring of the distal portion of the tendon to normal functioning tendon still its distal end is fixed to its insertion [2]. Tendons are strong enough to sustain the high tensile forces that result from muscle contraction during joint motion yet are sufficiently flexible to angulate around bone surfaces and to deflect beneath retinaculae to change the final direction of muscle pull. Living tissue, tendons are dynamic and change their mechanical properties in response to stress which leads to functional adaptation and optimal operation of the tissues [3]. Tendons appear to remodel in response to the mechanical demands placed on them; they become stronger and stiffer when subjected to increased stress and weaker and less stiff when the stress is reduced. Physical training has been 
found to increase the tensile strength of tendons [4].

The extrinsic extensors of the fingers and thumb are all innervated by the posterior interosseous nerve (PIN) branch of the radial nerve. The thumb has three separate extrinsic extensors, originate from the dorsal ulna in the midforearm and are innervated by the PIN. The abductor pollicis longus (APL) inserts on the radial base of the thumb MC to produce some extension, but mostly abduction. The extensor pollicis brevis (EPB) inserts on the base of the thumb proximal phalanx, and the extensor pollicis longus (EPL) inserts on the base of the thumb distal phalanx [5].

Extension is the movement of the thumb in a lateral or coronal plane away from the palm as to maintain the plane of the thumbnail at right angles to the plane of the other fingernails, movement occurs between the trapezium and the first metacarpal bone, at the metacarpophalangeal and interphalangeal joints, produced by the extensor pollicis longus and brevis. Abduction is the movement of the thumb in an anteroposterior plane away from the palm, the plane of the thumbnail being at right angles to the plane of the other nails movement occurs mainly between the trapezium and the first metacarpal bone; a small amount of movement occurs at the metacarpophalangeal joint, produced by the abductor pollicis longus and brevis [6].

In spastic cerebral palsy, dynamic approach to thumb-in-palm deformity. A, Release of adduction contracture through Z-plasty first web incision B, Transfer of PL to EPL, which has been released from third dorsal compartment. C, Transfer of distal portion of tendon of EPL to PL end to side, so- called dynamic tenodesis. If there is no suitable donor for active transfer, divide the APL tendon and reroute its distal portion volarly, attaching it in an end-to-side fashion to the flexor carpi radialis tendon under sufficient tension to maintain metacarpal abduction, and provides a dynamic abductor tenodesis. If the flexion deformity at the metacarpophalangeal joint is significant but joint stability is normal, a similar tenodesis of the extensor pollicis brevis tendon may be performed, don't create a disabling hyperextension deformity at MCP joint [7].

Tendon transfer to augment thumb extension should be performed after good passive motion is attained; it is contraindicated when there is weak pinch, correction of contracture is the essential principle as maximum passive motion of all joints must be present before tendon transfer is performed because no tendon transfer can move stiff joint and it is impossible for a joint to have more active motion postoperatively than it had passively preoperatively, adequate strength and appreciation of the relative strength of the forearm muscles is important in selecting an appropriate motor donor [8].

Boyes stringing of EPL: Tsuge noted of bow stringing EPL across radial aspect of the wrist was prevented by hooking it around the insertion of APL. Amplitude of motion of tendon excursion for each muscle, Boyes had suggested the use of the following values for practical purposes: Finger extensors and EPL: $50 \mathrm{~mm}$, Finger flexors: $70 \mathrm{~mm}$. These numbers of critical importance and significance since it is impossible for wrist flexors with an excursion of $33 \mathrm{~mm}$ to substitute fully of finger extensors with an amplitude of $50 \mathrm{~mm}$, the true amplitude of the tendon cannot be increased. Two things can be done to augment its effective amplitude: Firstly, a muscle converted from monoarticular to biarticualr or multiarticular therapy effectively utilizing the natural tenodesis effect. Second factor is extensive dissection of the muscle, surrounding facial attachments (9). Timing of tendon transfer after radial nerve sufficient time is determined by using Seddons figures for nerve regeneration is approximately $1 \mathrm{~mm}$ per day, this means 5 or 6 months before PR and ACRL return following nerve repair and remaining muscles 7.5 months [9] (Table 1).

Table1. Work capacity of forearm muscle (Boyes, 1964) (9).

\begin{tabular}{|l|l|l|l|}
\hline \multicolumn{2}{|l|}{ Donor muscles (MKg) } & \multicolumn{2}{l|}{ Recipient muscles (MKg) } \\
\hline BR & 1.9 & EPL & 0.1 \\
\hline PT & 1.2 & APL & 0.1 \\
\hline FCR & 0.8 & EPB & 0.1 \\
\hline FCU & 2 & EDC & 1.7 \\
\hline PL & 0.1 & EIP & 0.5 \\
\hline FDS & 4.8 & ECRL & 1.1 \\
\hline FDP & 4.5 & ECRB & 0.9 \\
\hline FPL & 1.2 & ECU & 1.1 \\
\hline
\end{tabular}

\section{Patients And Methods}

The procedure was done in 30 patients divided into 3 groups according to the etiology of deficient extension and abduction of the thumb. 12 patients with irreparable radial nerve palsy and 8 patients of brachial plexus palsy (C5-6-7) were in need to restore a combination of thumb extension and abduction, 10 patients 
with spastic cerebral palsy with 7 cases absent abduction and extension thumb and 3 cases of thumb in palm.

Three groups of patients was managed by active dynamic tenodesis of EPL.

Group 1: 12 cases of irreparable radial nerve injury. Age: from 6-25 years, sex: 4 female and 8 male (Figure 1).

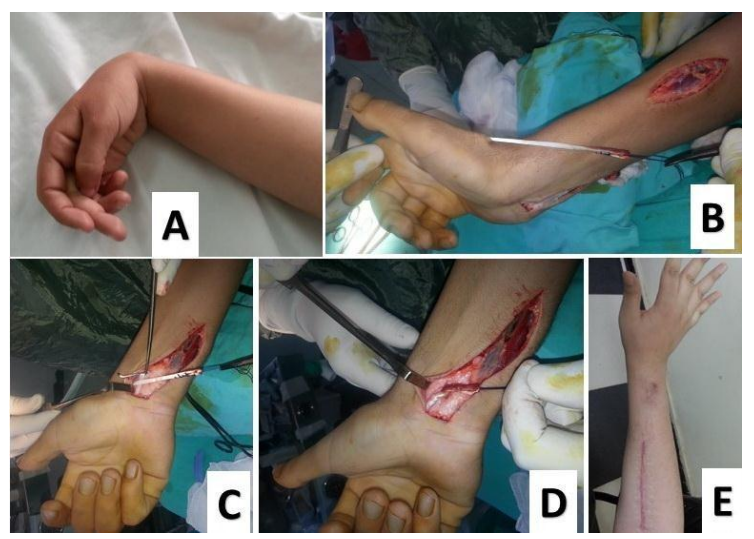

Figure1. Case N2 irreparable radial nerve injury A) Preoperative photo; $B$ ) intraoperative; $C \& D)$ postoperative photos after healing.

Group 2: (8 cases) of brachial plexus palsy $\mathrm{C}$ 5-6-7 neglected with absent MEMP in muscles of extensor pollicis brevis-longus and abductor pollicis. Age: from 4-40 years. Obstetric palsy 5 cases, sex: 2 female and 3 male. Traumatic Brachial Plexus palsy 3 cases, sex: 1 female and 2 male (Figure2).

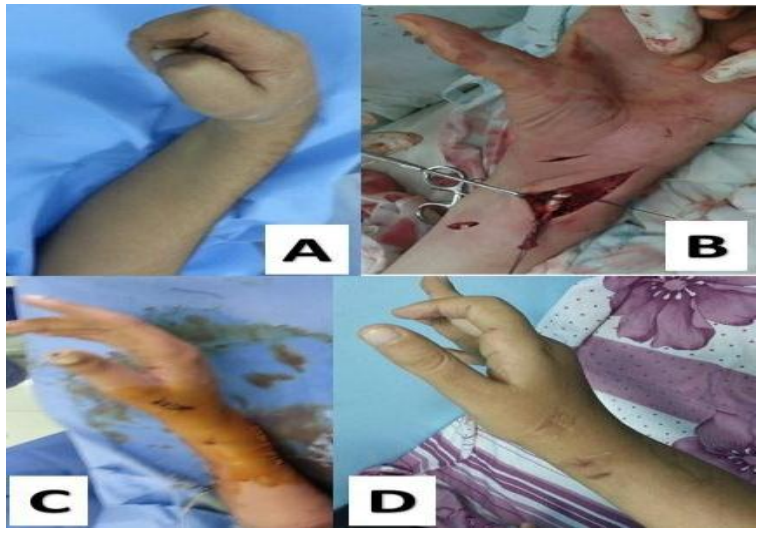

Figure2. Case No. 3: Traumatic brachial plexus palsy, K-wire Fixation to prevent hyperextension of MCP jo.

Group 3: (10 cases) of spastic cerebral palsy with 7 cases type 1 Sakellarides classification of thumb in palm and 3 cases type 3 thumb in palm. Age: from 4 to 18 years. Sex: 3 female and 7 male. IQ: $<80 \%$ spastic cerebral palsy unilateral disability. Type1 Sakellarides classification of thumb in palm (weak EPL and weak abduction of thumb treated by transferring of PL to EPL, 3 cases CP type 3 thumb in palm with need for first release of first dorsal web skin space, release of adductor pollicis, myotomy of flexor pollicis brevis and fractional lengthening of flexor pollicis longus to attain subtle mobile joints of the thumb, then the patient underwent extensive physiotherapy before our procedure is done (Figure 3 ).

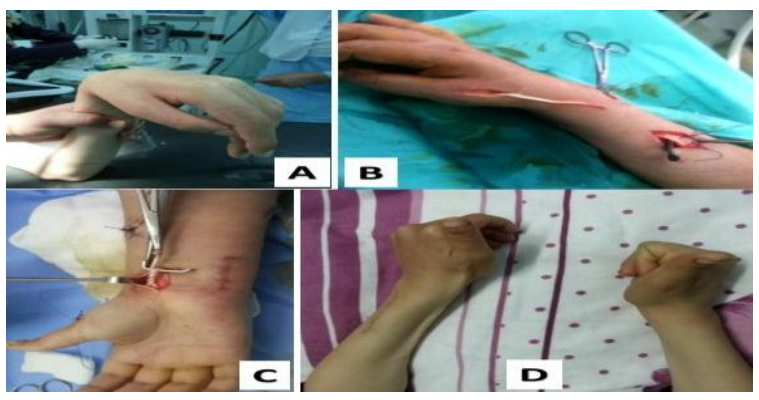

Figure3. Case No.1: Spastic cerebral palsy thumb in palm 2 stages procedure, second step reconstruction of EPL by PL.

\subsection{Operative Technique}

Extensor pollicis longus rerouting: EPL rerouting (Transfer EPL from third to first extensor compartment so extensor adductor to abductor. Redirect the EPL tendon along the radial aspect of the wrist, using redirecting the tendon through third compartment, the EPL tendon is moved from dorsal forearm and rerouted to the volar forearm the EPL can be routed around the EPB and APL tendons just proximal to the compartment to fixed and wrapped around the normal PL to achieve active tenodesis of EPL as PL was still attached to its normal anatomical tendon to its deep layer to the flexor retinaculum and its superficial layer to palmar aponeurosis which creates a combination of abduction and extension force and tenodesis on the thumb Identify the extensor pollicis longus at the metacarpophalangeal joint, and dissect it out from the extensor aponeurosis for a distance of $10 \mathrm{~mm}$ distal to the joint. This leaves a longitudinal defect $4 \mathrm{~mm}$ wide in the extensor hood. Identify EPL through a longitudinal incision at the distal radius, and withdraw it into the forearm.

The EPL is located at Lister's tubercle through a dorsal longitudinal incision, the retinaculum ulnar to the tubercle is released, allowing the thumb EPL is moved from Lister's canal after the tendon is cut at myotindinous junction, in most cases we don't release the canal just pull distal end of the EPL tendon is driven from the wound, small longitudinal volar incision on the ulnar side of the PL tendon, the PL tendon is 
identified, the EPL tendon is mobilized to be sutured to the fixed non-detached PL by EPL tendon is wrapped around the PL tendon, the suturing started at distal end of the PL to prevent cut through of tendon, fix tendon in tension position of thumb in fully extended and abducted with wrist in neutral position, .If the metacarpophalangeal joint is hyper extensible, transverse tunnel in capsule of MCP should be placed proximal to the articular surface to prevent further hyperextension. In this situation, a temporary Kirschner wire should be inserted across the slightly flexed metacarpophalangeal joint. We believe that procedure is simple, but the indication for surgery requires mature judgment and a thorough evaluation of the whole patient.

\section{Postoperative Management}

Long arm splint is applied which immobilizes the forearm in 15-30 degree pronation, the wrist in 45 degree dorsiflexion, $\mathrm{MB}$ joints in slight flexion $\left(10-15^{\circ}\right)$ and the thumb in maximum abduction and extension. The splint and sutures are changed and removed 14 days then forearm wrist splint cast is done for 4 weeks, the cast is removed after 6 weeks. In a planned exercise program, the patient is instructed to do synergistic movements, a well-motivated patient should have good control of function of 3 months and many patients take a long time 6 months to maximum recovery. At 3 weeks after cast removal all light activities of daily living are encouraged including those with pinch and grasp. Three months of postoperative therapy is recommended. The initial postoperative therapy goal is to have the patient actively attempt to inhibit any thumb adduction during proximal exercises. This is assessed and achieved before active extension and abduction firing is attempted, usually within 2 weeks after splint removal. Therapy then progresses to include light cylindrical grasp and opposed light pinch of small objects, with the focus on balanced palmar and radial abduction. Squeezing and tight pinch are avoided for the first 3 weeks of therapy to avoid thumb adduction pattern. If the patient is unable to inhibit adduction at least partially then squeezing and tight grasp are limited in the therapy program but are included as needed for activities of daily living.

\section{Results}

The follow up period was (9 month-48 month), one hundred percent of 30 patient had improved thumb function following active dynamic tenodesis for all case with 3 group of different etiology of deficient thumb extension and abduction.

Group I: 12 cases of irreparable radial nerve (9 cases fracture shaft humerus - 2 cases supracondylar fracture humerus -1 case schwannoma of radial nerve) we used " modified medical research council system [10] thumb abduction and extension was grade 4 in 5 patients with active movement against resistance with motion reaching more than $1 / 2$ normal range and grade 5 in 7 patients with normal power and range of motion. Functional result according to modified medical research council system good result means grade $4(41.7 \%)$ and full means grade $5(58.3 \%)$. All cases were satisfied so we considered good and full thumb function are satisfactory results.

Group II: We used in assessment of hand function and thumb function after reconstruction by dynamic tenodesis of $\mathrm{E} \mathrm{P} \mathrm{L} \mathrm{by} \mathrm{P} \mathrm{L}$ in brachial plexus palsy ( C 5-6-7) ( Hospital For Sick Children Muscle Grading System ) 5 cases with thumb abduction and extension was grade 6 with active movement against resistance with motion reaching more than $1 / 2$ normal range against gravity and 3 cases with thumb abduction and extension was grade 7 with active movement against resistance with full motion (11). Functional result according to Hospital For Sick Children Muscle Grading System, good result means grade $6(60.2 \%)$ and full means grade 7 ( $39.8 \%$ ). $100 \%$ of cases are satisfied so we considered good and full thumb function are satisfactory results.

Group III: We used in assessment of hand function and thumb function after reconstruction by dynamic tenodesis of EPL by PL in cases of spastic CP by using (House Scale Classification) (2) which depends on evaluating hand function of children with a thumb in palm deformity.

(House Scale Classification) used a nine point scale (0 to 8$)$ range from does not use hand (0), to passive use to active spontaneous hand use (8) . 8 cases of CP were score level 7 according to House Scale Classification, the patients used those hands spontaneously and partial activity level they perform bimanual activities easily and occasionally uses the hand spontaneously. 2 cases of $\mathrm{CP}$ were score level 8 
according to House Scale Classification, the patients used those hands spontaneously and completely and with maximum activity level, they perform bimanual activities easily and completely independent without reference to the other hand. Functional result according to (House Scale Classification), good result means level 7 (80\%) and full means grade 8 (20\%). $100 \%$ of cases are satisfied, so we considered good and full thumb functions are satisfactory results.

\section{DISCUSSION}

Surgical technique of Extensor pollicis longus re-routing as EPL passes around the lister's tubercle also has an adductor effect due to its path, radicalizing this route expected to have a positive effect on abduction.

Goldner et al removed EPL Tendon from its fibro - osseous channel, creating a new pulley over the radial styloid and moving the route of the tendon radially. Manske took the EPL tendon first dorsal retinucular so eliminating the need for a new pulley. Rayan and Saccone transected EPL tendon proximal to the retinaculum then anastomosed the distal EPL tendon taken through the first dorsal compartment with the proximal strip [2]. Ricado Monreal [12] stated restoration of thumb extension as a great problem in cases of plexus hand, in EPL tenodesis, radiodosal deviation of the wrist joint is inevitable and adduction of the thumb during dynamic tenodesis of finger extension but rerouting of EPL beneath APL tendon and fixing to PL end to side functions well, stabilizing both the wrist and thumb MP and IP joints [12].

In our study : The transected EPL tendon at myotindinous junction then remove the distal EPL tendon from its fibro-osseous tunnel then we reroute EPL around the EPB and APL tendons just proximal to the first extensor compartment so a new pulley is made to maintain the position of EPL and increase its abduction and extension action, then distal end of EPL is wrapped around PL tendon end to side of PL which still in its anatomical insertion in flexor retinaculum and palmar aponeurosis then tension of EPL in maximum thumb abduction and extension is maintained and palmaris longus tendon is tensioned by wrist extension and traction on the PL tendon this position is maintained by suture. In our Technique we avoid ischemic necrosis of the tendons by release of the third extensor compartment and EPL rerouted around EPB and APL and doesn't pass the tendon on the first compartment to avoid ischemic necrosis of the tendons secondary to post-operative edema.

\section{CONClusion}

Supporting thumb abduction and extension tendon was done by reinforcement which performed by dynamic tendon transfer of PL to EPL which rerouted around APL and EPB as a pulley and fixing the EPL to PL tendon at proper tension "tenodesis so we do dynamic active tenodesis of EPL " in deficient thumb abduction and extension so $100 \%$ of cases are satisfied, so we considered good and full thumb function are satisfactory results.

\section{REFERENCES}

[1] Scott L. Hansen, Mahesh M, David M. Young. Hand surgery. Current Surgical Diagnosis and Treatment. 2006; chapter 44.

[2] Ozkan T and Serdar T. Upper extremity surgery in spastic cerebral palsy. Surgery cerebral palsy. JAREM 2012, 2: 43-54.

[3] Ann Ebarr and Jane Bear: Biomechanics of the wrist and hand, Biomechanics of joint, part 2 basic Biomechanics of the musculoskeletal system, Margreta Nordun third edetion: Chapter (14) 359: 387-2001.

[4] Margreta Nordun, Tobias Lorenz: Biomechanics of tendons and ligaments, basic Biomechanics of the musculoskeletal system , third edition : Chapter (4) 103: 119-2001.

[5] Scott D. Lifchez, Subhro K. Sen. Surgery of the hand and wrist. Schwartz's Principles of Surgery. 2010; chapter 44.

[6] Richard S. Snell. The upper limb. Clinical Anatomy by Regions. 2007; 9: 480-520.

[7] C. A, Mmowery, R. H. Gelberman, C. E, Rhoads. Upper extremity tendon transfers in cerebral palsy: electromyographic and functional analysis. JPediatr Orthop. 1985; 5: 69-72.

[8] Mark Hoffer, MD : Cerebral Palsy - (tendon transfere to agument some thumb extension ) operative hand surgery, David P. Green , Second edetion chapter (8) volume (1) - 215: $226-1988$.

[9] David P. Green : Radial nerve palsy, oprative hand surgery, Second edetion chapter (37) volume (2) - 1479:1498 - 1988. 

and Extension

[10] A E Hardy: birth injuries of the brachial plexus palsy incidence and prognosis , $\mathrm{J}$ bone and joint surg, vol. 63- b, no. 1 ( 1981$): 98-101$

[11] Howard M Clarke, M M AL- Qattan , C Curtis " obstetrical brachial plexus palsy . hand clincs . nov . 1995,11 ( 4 ) : $563-581$.

[12] Ricado Monreal : dynamic tenodessis of the finger extensors to improve hand function after brachial plexus injury, amrican association for hand surgery , December 2010 , 5: 256 - 260.

Citation: Mohamed Abdelaziz MA Ghieth, Mohamed A Sebaai. Active Dynamic Tenodesis of Extensor Policis Longus by Palmaris Longus in Deficient Thumb Abduction and Extension. ARC Journal of Orthopedics. 2017; 2(1):1-6. doi:dx.doi.org/10.20431/2456-0588.0201001.

Copyright: (C) 2017 Authors. This is an open-access article distributed under the terms of the Creative Commons Attribution License, which permits unrestricted use, distribution, and reproduction in any medium, provided the original author and source are credited. 